\title{
The Effect of Integrity of Lateral Wall on the Quality of Reduction and Outcomes in Elderly Patients with Intertrochanteric Fracture: A Controlled Study
}

\author{
Hong-Li Deng $\mathbb{D}$, Yu-Xuan Cong, Hai Huang, Bin-Fei Zhang $\mathbb{D}$, Ya-Hui Fu, Jin-Lai Lei, \\ Hu Wang, Peng-Fei Wang, Yan Zhuang, and Chao Ke $\mathbb{D}$ \\ Department of Orthopedic Trauma, Honghui Hospital, Xi'an Jiaotong University, Xi'an, Shaanxi Province, China \\ Correspondence should be addressed to Chao Ke; 663701242@qq.com
}

Received 18 April 2021; Accepted 24 July 2021; Published 9 August 2021

Academic Editor: Panagiotis Korovessis

Copyright $\odot 2021$ Hong-Li Deng et al. This is an open access article distributed under the Creative Commons Attribution License, which permits unrestricted use, distribution, and reproduction in any medium, provided the original work is properly cited.

\begin{abstract}
Objective. The study is aimed at evaluating the effect of the integrity of lateral wall on the quality of reduction and outcome in intertrochanteric fracture treated with proximal femoral nail antirotation (PFNA). Methods. Medical record systems for elderly patients with intertrochanteric fracture treated with PFNA were included. The patients were divided into incompetent and intact lateral wall groups. Patients' baseline characteristics, quality of reduction, and Harris Hip scores (HHS) were collected. Results. The study included 115 patients with intertrochanteric fractures, with 59 in the incompetent lateral wall group and 56 in the intact group. Lateral wall thickness was $16.47 \pm 2.46 \mathrm{~mm}$ and $23.68 \pm 1.59 \mathrm{~mm}$ in the incompetent group and intact group $(t=-18.766, P<0.001)$, respectively. There was no significant difference in the quality of reduction $(P=0.646)$ between intact and incompetent groups. Mean HHS at final follow-up were $83.02 \pm 13.89$ in the incompetent group and $86.04 \pm 3.39$ in the intact group, with no significant difference $(P=0.123)$. In addition, there was no significant difference in weight-bearing or clinical healing between intact and incompetent groups. The partial weight-bearing with crutches was allowed at $2.71 \pm 0.93$ and $2.66 \pm 1.01$ weeks after the operation in the incompetent and intact groups. Time to clinical healing was $5.83 \pm 0.99$ and $6.00 \pm$ 0.92 months in the incompetent and intact groups, respectively. However, the operative time in the incompetent group (58.54 \pm 18.14 mins) were longer than that in the intact group $(51.79 \pm 17.77$ mins). Conclusions. In conclusion, it seems that lateral wall thickness does not affect the quality of reduction and outcome in patients with intertrochanteric fracture receiving PFNA.
\end{abstract}

\section{Introduction}

Geriatric intertrochanteric fractures represent an increasing public health problem all over the world. According to recent epidemiological investigation, the incidence rates of intertrochanteric fractures were $171 / 100,000$ and have been kept increasing recently [1].

The trochanteric lateral wall is primarily the lateral femoral cortex of the drilling site for head-neck fixation implant [2]. Describing in Hsu's study, the lateral wall thickness is the distance in millimeters from a reference point $3 \mathrm{~cm}$ below the innominate tubercle of the greater trochanter, angled $135^{\circ}$ upward to the fracture line on the anteroposterior radiograph [3]. This criterion has been accepted and included in the 2018 version of AO/OTA Fracture and Dislocation Classification Compendium [4]. If the thickness is less than $20.5 \mathrm{~mm}$, the lateral wall is divided into incompetent subtype.

The intact lateral wall plays a crucial role in the stabilization of intertrochanteric fracture. Under the implant of extramedullary fixation, an intact lateral wall could provide good biomechanical support [5, 6]. However, intramedullary nailing is a good option, especially when lateral wall is incompetent [7]. Therefore, the integrity of the lateral wall in intertrochanteric fractures plays an essential role in choosing the type of implant. In the past, the proximal femoral locked compression plate was recommended for use in fixation of complex proximal femur fractures such 
as A3 intertrochanteric fracture in AO/OTA classification version 2007 [8]. However, rates of failure after fixation using plate for those fractures were reported as $44 \%$ [9], and the reoperation rate $(4 \%)$ after surgery with intramedullary nailing was significantly lower than that after surgery with sliding hip screw [10]. In addition, the strength of lateral wall in elderly people is so weak, and the preoperatively intact lateral wall may be broken during the operation when using plate in some patients. Under these views, some surgeons prefer intramedullary nails in all types of intertrochanteric fractures.

The intact lateral wall takes about $67 \%$ of all intertrochanteric fractures, and incompetent lateral wall takes 33\% [11]. Duration of the operation, intact lateral wall demonstrates the simple fracture lines, and it is easy to reduce. In contrast, incompetent lateral wall is usually difficult to achieve good reduction because of multifragmentary fractures. Even though the new AO/OTA classification was introduced in 2018, there are no related studies involving the quality of reduction and prognosis affected by the lateral wall. Thus, the present study is aimed at evaluating the effect of the integrity of lateral wall on the quality of reduction and outcome in intertrochanteric fracture treated with intramedullary nails.

\section{Materials and Methods}

2.1. Patients including. The Ethics Committee of the Xi'an Honghui Hospital approved the study (No. 2020064). The inclusion criteria were (1) age $\geq 65$ years; (2) hip pain, tenderness, dysfunction, ecchymosis, and local swelling; (3) injury from falling from a height, slipping, traffic accident, or other; (4) unilateral intertrochanteric fractures were confirmed using radiography, and the integrity of lateral wall could be distinguished; (5) operative treatment of closed reduction and internal fixation was undergone by proximal femoral nail antirotation (PFNA); and (6) at least six months of follow-up.

The exclusion criteria were (1) age $<65$ years, (2) multiple injuries with intertrochanteric fractures and other fractures and only operation for intertrochanteric fractures, (3) severe comorbidities and could not suffer the operation, and (4) the patients received the extramedullary hip screws. We searched the medical system records for patients with intertrochanteric fractures. The patient record search periods were from January 2018 to June 2019. In Figure 1, when the thickness $(d)$ was less than $20.5 \mathrm{~mm}$, it was divided into incompetent group; when $(d)$ was longer than $20.5 \mathrm{~mm}$, it was divided into intact group.

2.2. Surgical Strategy. The surgical strategy is similar to the article from $\mathrm{Ma}$ et al. [12]. Upon admission of patients with intertrochanteric fractures, the blood routine test and other blood samples were examined to assess the hidden blood loss. When patients were in stable condition, we performed the operation as soon as possible. All procedures were performed under general anesthesia by the same team.

Duration the operation, the C-arm fluoroscopy was used to check the reduction and the procedure of inserting the

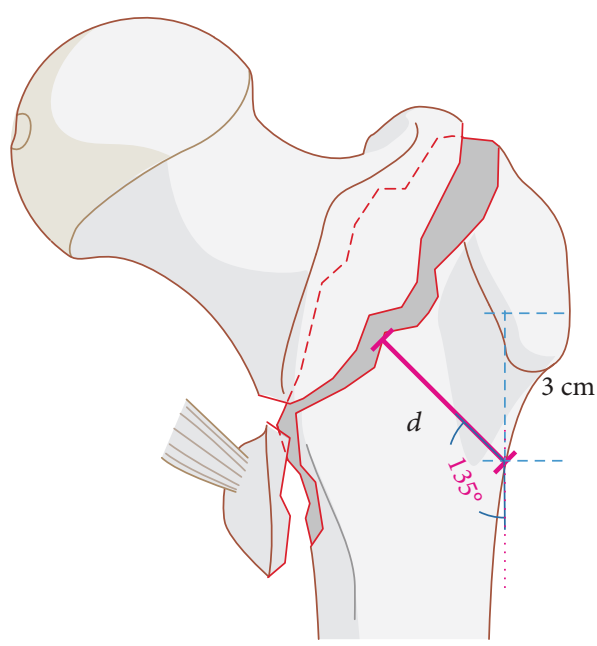

FIgURE 1: The schematic diagram of the intertrochanteric lateral wall.

nail. We used the anterior-posterior and lateral views of the hip to assess the quality of reduction. We tried the closed reduction firstly. If the quality is poor, there would be another reduction. Once the reduction was acceptable, we used the guide needle to locate the insertion point by puncturing the skin. After reconfirming the fracture reduction, we cut a $5 \mathrm{~cm}$ incision along the direction of the guide needle. The proximal femur was gradually reamed, and then, a nail was implanted. After adjusting the height of the nail, the frame to implant the head screw was fixed. After inserting the needle and measuring the length of the head screw and reaming, the head screw was implanted and compressed the fracture. Then, a distal locking screw was implanted under the directing frame, and a tail screw of the nail was locked. Finally, we washed out and closed the incision.

2.3. Follow-Up. After discharging, the patients were recommended to perform isometric exercises in bed as soon as possible and allowed partial weight-bearing at 2-4 weeks postoperatively. The surgeons determined the timing of full weight-bearing according to fracture healing. The patients returned to the hospital at least once a month for the first six months postoperatively, and an X-ray was used to evaluate the fracture union.

2.4. Outcomes. The outcomes were quality of reduction, Harris Hip scores (HHS), operative time, intraoperative blood loss, blood transfusion, intraoperative liquid, follow-up time, clinical healing time, weight-bearing time, and complications (deep vein thrombosis, wound infection, revision, mortality). Chang's reduction quality criteria was used as the tool to assess the quality of reduction $[13,14]$.

2.5. Statistical Analysis. Statistical analysis was performed using SPSS version 25.0 (SPSS Inc., Chicago, IL, USA). We assessed whether measurement data were normally distributed using the Shapiro-Wilk test and then using independent-samples $t$-tests. For frequency data, the chi- 
square or Fisher's exact test was used. If $P$ was $<0.05$, there was the considered statistically significant difference.

\section{Results}

3.1. Clinical Characteristics. This study included 115 patients who suffered intertrochanteric fractures. There were 82 females and 33 males. The lateral wall incompetent group included 59 patients, and the intact group included 56 patients admitted between January 2018 and June 2019. The same team performed all 115 operations. The mean age was 80.07 years in the incompetent group and 80.63 years in the intact group, respectively. Mechanisms of injury included slipped, high falling, accident, and others. The most common mechanism was slipped, which occurred in $81.35 \%$ and $89.28 \%$ of patients in incompetent and intact groups, respectively. Preoperative Visual Analogue Scale (VAS) scores were $3.86 \pm 0.99$ and $4.25 \pm 1.15$ in the incompetent and intact groups, respectively.

Lateral wall thickness was $16.47 \pm 2.46 \mathrm{~mm}$ and $23.68 \pm$ $1.59 \mathrm{~mm}$ in the incompetent group and intact group $(t=-18.766, P<0.001)$, respectively. The intertrochanteric fractures were divided into three subgroups according to the 2007 AO/OTA classification, and there were 1, 28, and 30 of the incompetent group and 23,17, and 16 of the intact group in $31 \mathrm{~A} 1.3,31 \mathrm{~A} 2.2$, and $31 \mathrm{~A} 2.3$ subgroups, respectively. We found a statistically significant difference in fracture types between the two groups $\left(\chi^{2}=27.057, P<0.001\right)$. The percentage of $31 \mathrm{~A} 1.3(41 \%)$ in the intact group was more than the incompetent group (1.6\%). In addition, there were no significant differences in the comorbidities (hypertension, diabetes, stroke, and associated injuries) between the two groups. The time from injury to admission was $0.97 \pm 0.83$ and $0.86 \pm 0.86$ days in the incompetent group and intact group, respectively. The time from admission to operation was $2.53 \pm 0.95$ days and $2.27 \pm 0.86$ days in an incompetent and intact group. The length of stay in hospital in the incompetent group $(7.39 \pm 3.40$ days $)$ was similar to the intact group (6.52 \pm 2.35 days). Detailed baseline information is shown in Table 1.

3.2. Comparison of Operative Characteristics. Mean operative time were $58.54 \pm 18.14 \mathrm{mins}$ and $51.79 \pm 17.77 \mathrm{mins}$ in the incompetent and intact groups, respectively. The operative time in the incompetent group was longer than that in the intact group $(t=2.016, P=0.046)$. However, the blood transfusion in the incompetent group $(1.31 \pm 1.16 \mathrm{U})$ was similar to that in the intact group $(1.29 \pm 1.11 \mathrm{U}, P=0.908)$. The intraoperative blood loss was $64.75 \pm 19.51 \mathrm{ml}$ in the incompetent group and $68.21 \pm 22.89 \mathrm{ml}$ in the intact group, without significant differences. Also, there was no significant difference in the intraoperative liquid between the two groups $(P=0.059)$, incompetent group $(1305.08 \pm 302.53$ $\mathrm{ml})$ versus intact group (1203.57 $\pm 266.26 \mathrm{ml}$; Table 2$)$.

3.3. Follow-Up and Fracture Healing. Follow-up time was not significantly different between the two groups $(10.98 \pm 4.32$ months in the incompetent group and $11.13 \pm 3.92$ months in the intact group; $P=0.854)$. During the follow-up, we assessed the time to postoperative weight-bearing and time to clinical healing. The partial weight-bearing with crutches was allowed at $2.71 \pm 0.93$ and $2.66 \pm 1.01$ weeks after the operation in the incompetent and intact groups. We evaluated clinical healing based on radiographic findings, symptoms, and signs. Time to clinical healing was $5.83 \pm 0.99$ and $6.00 \pm 0.92$ months in the incompetent and intact groups, respectively. We found no significant differences between the two groups in the time to weight-bearing or clinical healing (Table 2). The patient with an incompetent lateral wall is shown in Figure 2, and with intact lateral wall is shown in Figure 3.

3.4. Functional Outcomes and Quality of Reduction. Mean HHS at final follow-up were $83.02 \pm 13.89$ in the incompetent group and $86.04 \pm 3.39$ in the intact group, with no significant difference $(P=0.123$; Table 2$)$. The quality of reduction was assessed after the operation by $\mathrm{X}$-ray view. The quality was divided into three levels: excellent, acceptable, and poor. In the incompetent group, the excellent level has taken $54 \%$ of all, and the acceptable level has taken $42 \%$ of all. In the intact group, the excellent level has taken $62 \%$ of all, and the acceptable level has taken $34 \%$ of all. There were no significant differences in the distribution $(P=0.646$; Table 2$)$. The four patients who suffered a poor reduction were classified into $31 \mathrm{~A} 2.3$ types.

3.5. Postoperative Complications. We assessed the deep vein thrombosis, superficial infection, revision, and mortality after the operation (Table 2). No deaths occurred during the hospital stay and following-up. In the incompetent and intact groups, 22 and 23 patients developed into deep vein thrombosis, respectively, with no significant difference in frequency $(P=0.678)$. One patient had the superficial infection in the intact group, and the patient was treated with antibiotics and wound care, and the infections ultimately healed. None of the patients experienced fixation failure needing revision during follow-up.

\section{Discussion}

The 2018 Compendium described it on the anteroposterior $\mathrm{X}$-ray measuring the lateral wall height to identify the intact and incompetent subtypes [4]. In essence, the role of the mechanical buttress of the lateral wall is primarily played by the anterior or anteromedial cortex [15].

The present study is aimed at evaluating the quality of reduction and outcome in intertrochanteric fracture with intact and incompetent lateral wall. The results show that (1) there was no significant difference in the quality of reduction between in intact and incompetent group, (2) mean HHS at final follow-up was comparable in two groups, (3) the mean operative time in the incompetent group was longer than that in the intact group, and (4) there were no significant differences between the two groups in the time to weight-bearing or clinical healing.

In the distribution of fracture types, the percentage of $31 \mathrm{~A} 1.3$ in the intact group was more than the incompetent group. This difference was depending on the characteristics 
TABLE 1: The characteristics in lateral wall incompetent group and lateral wall intact group.

\begin{tabular}{|c|c|c|c|c|c|}
\hline & Lateral wall incompetent group & Lateral wall intact group & Total & $t / \chi^{2}$ & $P$ \\
\hline No. of patients & 59 & 56 & 115 & & \\
\hline Age & $80.07 \pm 7.79$ & $80.63 \pm 6.14$ & $80.34 \pm 7.01$ & -0.427 & 0.670 \\
\hline \multicolumn{6}{|l|}{ Sex } \\
\hline Female & 38 & 44 & 82 & \multirow{2}{*}{2.817} & \multirow{2}{*}{0.093} \\
\hline Male & 21 & 12 & 33 & & \\
\hline \multicolumn{6}{|l|}{ Mechanism of injury } \\
\hline Slipped & 48 & 50 & 98 & \multirow{4}{*}{2.831} & \multirow{4}{*}{0.418} \\
\hline High falling & 3 & 2 & 5 & & \\
\hline Accident & 5 & 1 & 6 & & \\
\hline Other & 3 & 3 & 6 & & \\
\hline Preoperative VAS & $3.86 \pm 0.99$ & $4.25 \pm 1.15$ & $4.05 \pm 1.08$ & -1.924 & 0.057 \\
\hline Lateral wall thickness (mm) & $16.47 \pm 2.46$ & $23.68 \pm 1.59$ & $19.98 \pm 4.17$ & -18.766 & $<0.001$ \\
\hline \multicolumn{6}{|l|}{ AO/OTA classification (2007) } \\
\hline A1.3 & 1 & 23 & 24 & & \\
\hline A2.2 & 28 & 17 & 45 & 27.057 & $<0.001$ \\
\hline A2.3 & 30 & 16 & 46 & & \\
\hline \multicolumn{6}{|l|}{ Comorbidities } \\
\hline Hypertension & 25 & 29 & 54 & 1.022 & 0.312 \\
\hline Diabetes & 24 & 17 & 41 & 1.334 & 0.248 \\
\hline Stroke & 20 & 17 & 37 & 0.165 & 0.685 \\
\hline Associated injuries & 5 & 6 & 11 & 0.167 & 0.683 \\
\hline Days from injury to admission (days) & $0.97 \pm 0.83$ & $0.86 \pm 0.86$ & $0.91 \pm 0.84$ & 0.691 & 0.491 \\
\hline Days from admission to operation (days) & $2.53 \pm 0.95$ & $2.27 \pm 0.86$ & $2.40 \pm 0.92$ & 1.515 & 0.132 \\
\hline Length of stay in hospital (days) & $7.39 \pm 3.40$ & $6.52 \pm 2.35$ & $6.97 \pm 2.96$ & 1.592 & 0.114 \\
\hline
\end{tabular}

TABLE 2: The primary and secondary outcomes between two groups.

\begin{tabular}{|c|c|c|c|c|c|}
\hline & $\begin{array}{l}\text { Lateral wall incompetent } \\
\text { group }(n=59)\end{array}$ & $\begin{array}{l}\text { Lateral wall intact } \\
\text { group }(n=56)\end{array}$ & Total & $t / \chi^{2}$ & $P$ \\
\hline Operative time (mins) & $58.54 \pm 18.14$ & $51.79 \pm 17.77$ & $55.34 \pm 18.26$ & 2.016 & 0.046 \\
\hline Intraoperative blood loss (ml) & $64.75 \pm 19.51$ & $68.21 \pm 22.89$ & $66.49 \pm 21.29$ & -0.876 & 0.383 \\
\hline Blood transfusion $(\mathrm{U})$ & $1.31 \pm 1.16$ & $1.29 \pm 1.11$ & $1.30 \pm 1.13$ & 0.116 & 0.908 \\
\hline Intraoperative liquid (ml) & $1305.08 \pm 302.53$ & $1203.57 \pm 266.26$ & $1255.26 \pm 289.98$ & 1.906 & 0.059 \\
\hline Follow-up time (months) & $10.98 \pm 4.32$ & $11.13 \pm 3.92$ & $11.11 \pm 4.09$ & -0.184 & 0.854 \\
\hline Weight-bearing time (weeks) & $2.71 \pm 0.93$ & $2.66 \pm 1.01$ & $2.69 \pm 0.97$ & 0.282 & 0.778 \\
\hline Clinical healing time (months) & $5.83 \pm 0.99$ & $6.00 \pm 0.92$ & $5.92 \pm 0.95$ & -0.955 & 0.342 \\
\hline \multicolumn{6}{|l|}{ Complications } \\
\hline Deep vein thrombosis & 22 & 23 & 45 & 0.173 & 0.678 \\
\hline Superficial infection & 0 & 1 & 1 & 0.001 & 0.979 \\
\hline Revision & 0 & 0 & 0 & & \\
\hline Mortality & 0 & 0 & 0 & & \\
\hline HHS scores & $83.02 \pm 13.89$ & $86.04 \pm 3.39$ & $84.47 \pm 10.53$ & -1.554 & 0.123 \\
\hline \multicolumn{6}{|l|}{ Quality of reduction } \\
\hline Excellent & 32 & 35 & 67 & & \\
\hline Acceptable & 25 & 19 & 44 & 0.875 & 0.646 \\
\hline Poor & 2 & 2 & 4 & & \\
\hline
\end{tabular}




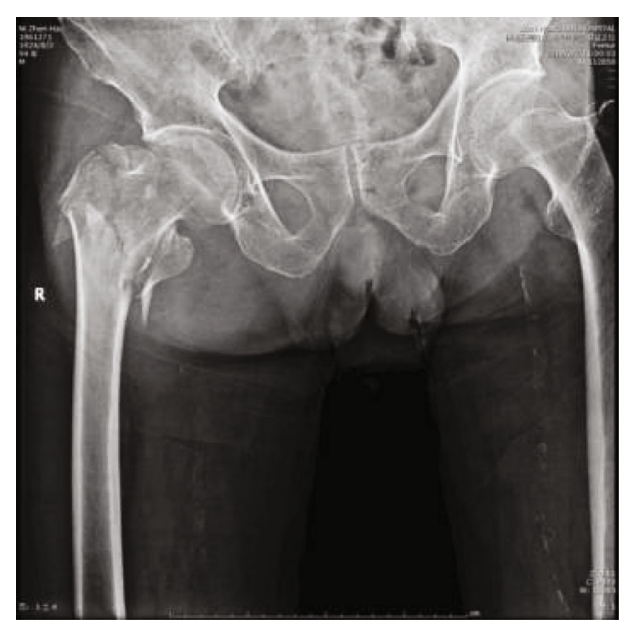

(a)

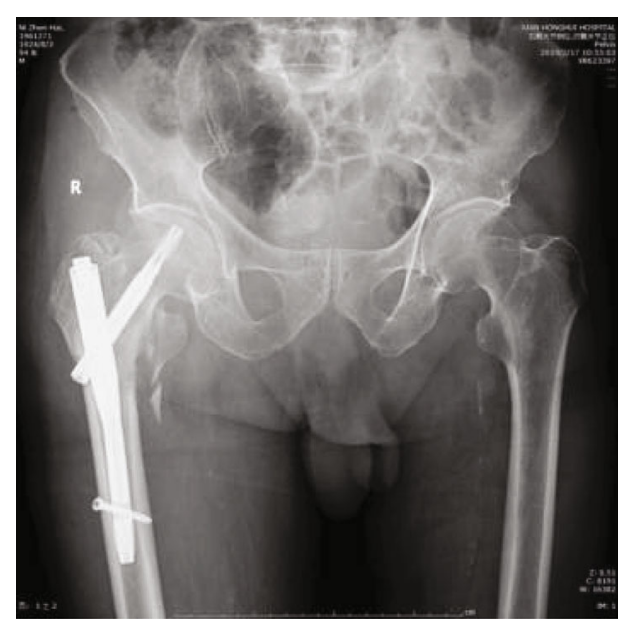

(c)

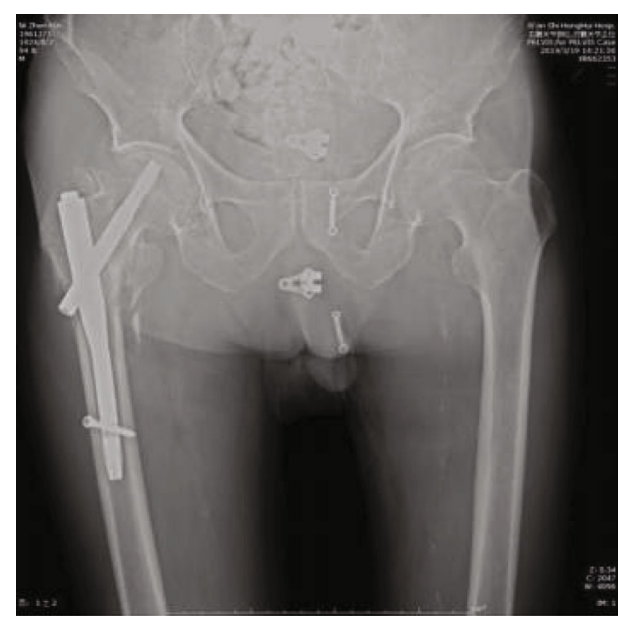

(e)

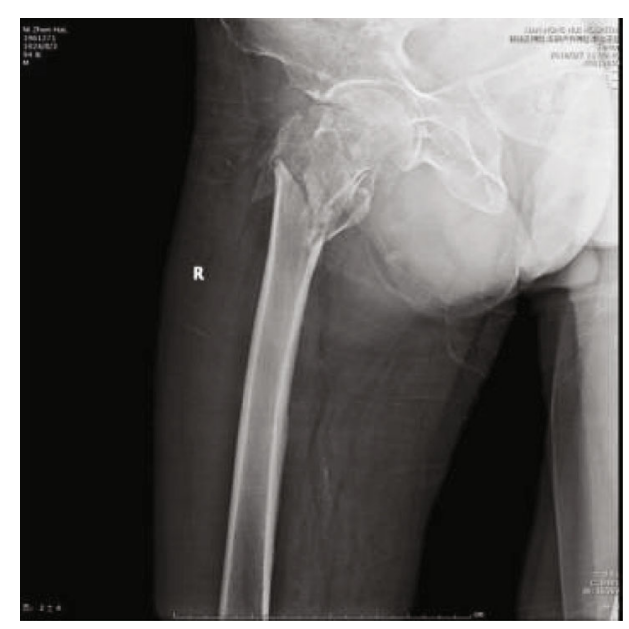

(b)

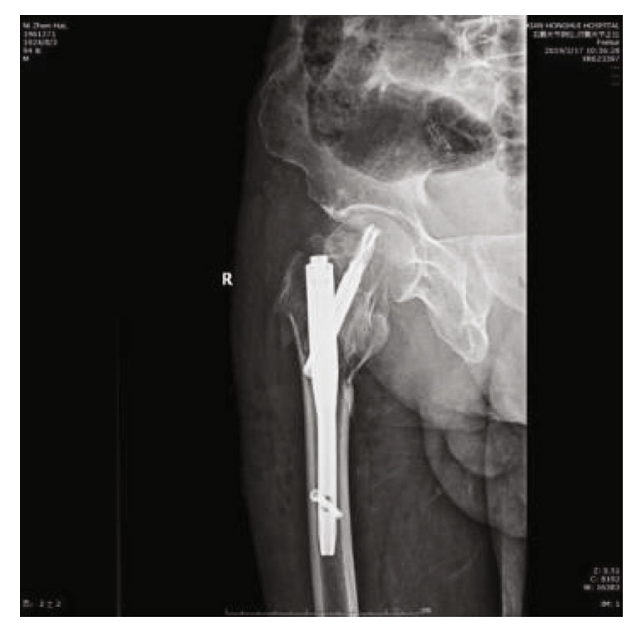

(d)

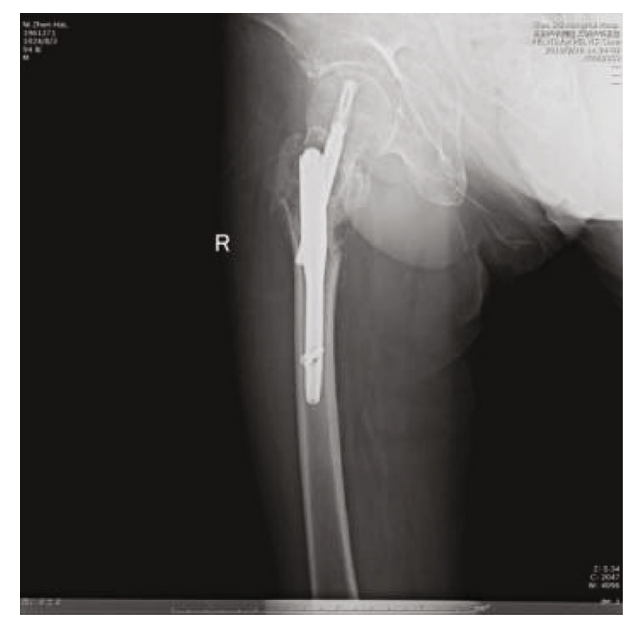

(f)

FIGURE 2: The patient receiving PFNA with incompetent lateral wall: (a) preoperative anterior-posterior view; (b) preoperative lateral view; (c) postoperative anterior-posterior view; (d) postoperative lateral view; (e) postoperative 3 months anterior-posterior view; (f) postoperative 3 months lateral view.

of the integrity of lateral wall and the classification of version 2018 [4]. In this study, considering the unstable intertrochanteric fractures and the necessity of intramedullary fixation and the possibility of intraoperative or postoperative lateral wall fracture when using the extramedullary plates $[7,16]$, we restricted the included population as 


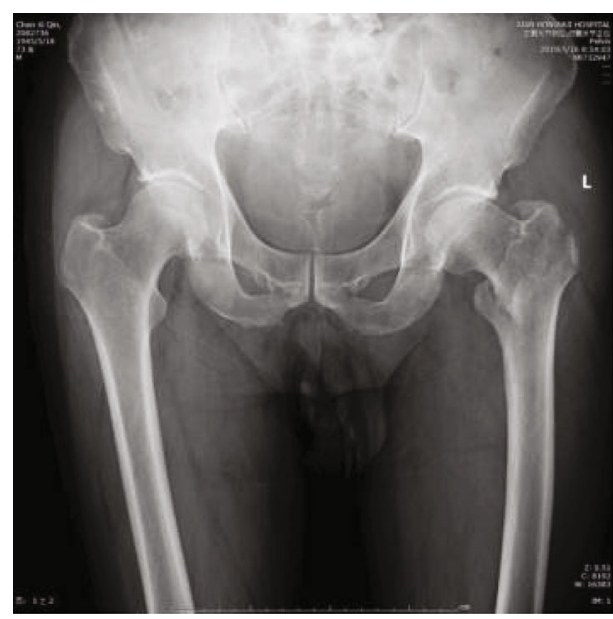

(a)

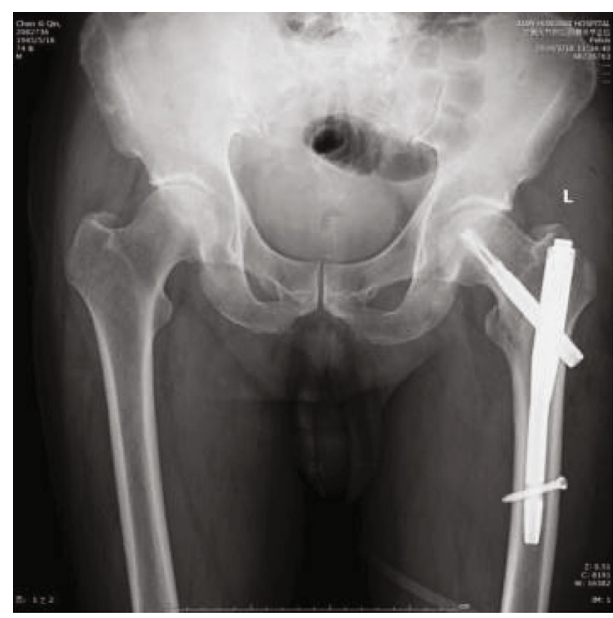

(c)

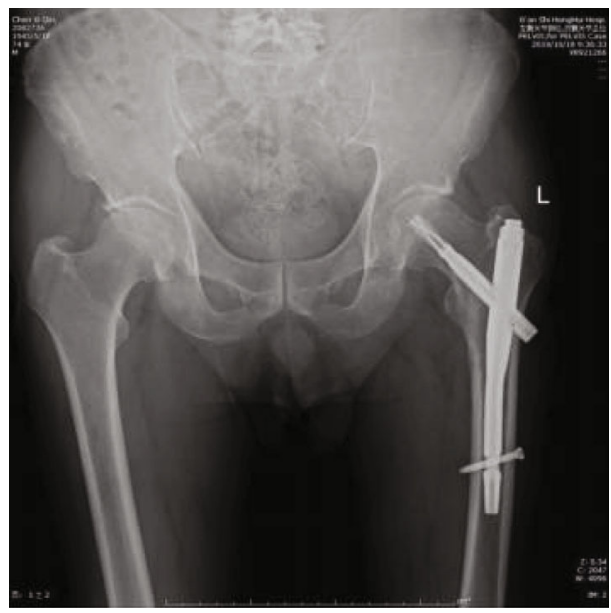

(e)

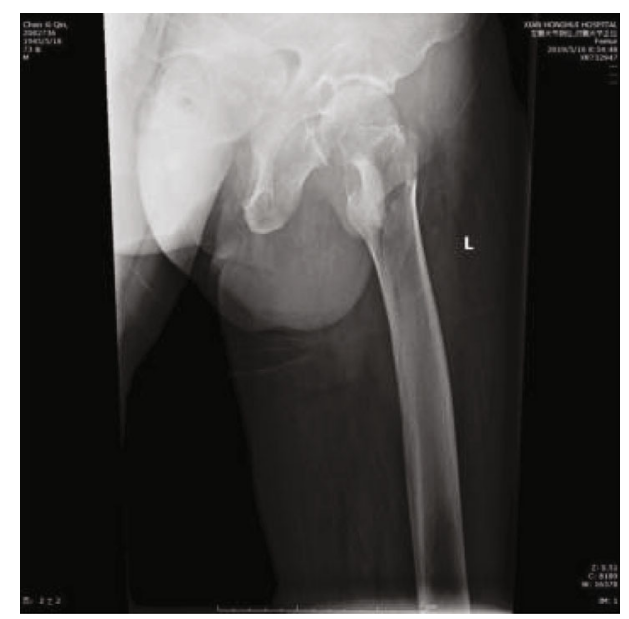

(b)

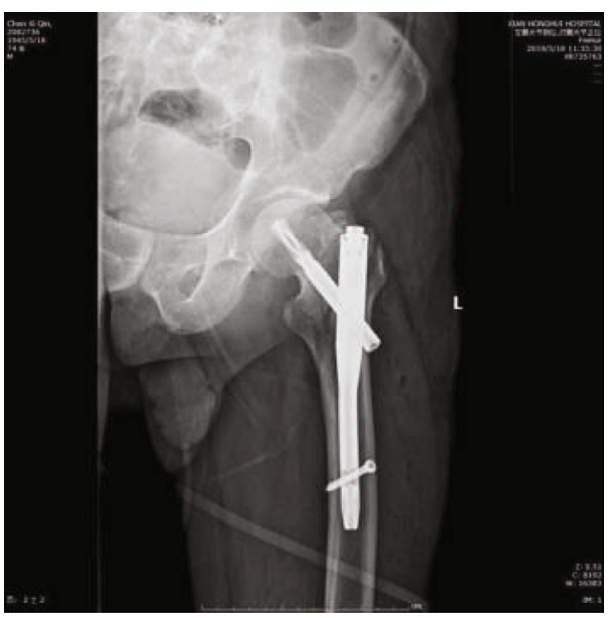

(d)

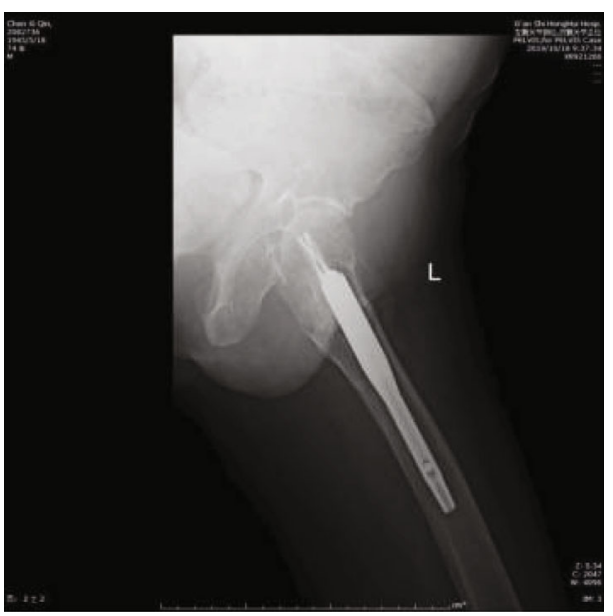

(f)

FIGURE 3: The patient receiving PFNA with intact lateral wall: (a) preoperative anterior-posterior view; (b) preoperative lateral view; (c) postoperative anterior-posterior view; (d) postoperative lateral view; (e) postoperative 4 months anterior-posterior view; (f) postoperative 4 months lateral view.

the receiving PFNA treatment to reduce the performance bias.

To our knowledge, this study is the first retrospective research focusing on the quality of reduction in intertrochan- teric fractures. When the quality levels were divided into excellent, acceptable, and poor, we found that the excellent and acceptable levels taken $96 \%$ of all in the incompetent and intact groups, respectively. The assessing of reduction 
was used Chang's criterion, with a good receiver operating characteristic curve and 0.87 area under the curve [13, 14]. Two chief orthopedic surgeons assessed it independently, and the third chief surgeon would participate in assessment when encounter disagrees.

When to HHS comparison, it was comparable in two groups. The patients we included in the study were more than 65 years, and the mean HHS was 84.47. In a study from Korea [17], the HHS at 6 months is 88.33-90.68 in an average of 76 years. Overall, the function in these patients was good.

In the quality of reduction and HHS, we did not find the differences between incompetent and intact lateral wall groups. However, there is a trend that mean HHS in the intact group $(86.04 \pm 3.39)$ was higher than the incompetent group $(83.02 \pm 13.89)$, and the proportion of excellent level in the intact group (62\%) was more than the incompetent group (54\%), without the statistically significant difference.

In this study, the mean operative time in the incompetent group was longer than that in the intact group. Duration the operation, all the patients received the closed reduction. In the incompetent group, we often use one or two small incisions to assist in reducing with periosteal elevator or 90-degree pliers. Once the reduction was acceptable, we often choose a short $170 \mathrm{~mm}$ intramedullary nail, and the procedure of inserting the nail is the same in everyone patient. Thus, we consider that the difference in operative time is from the process of reduction, especially in the assisted incision in the incompetent group. In a prospective study, the mean operative time is $\mathbf{5 7 . 2 5}$ mins, which is similar to our result [17]. Corresponds to the operative time, the fluoroscopy time in the incompetent group is more than that in the intact group [17]. We did not compare this index in the study.

In addition, there were no significant differences between the two groups in the time to weight-bearing or clinical healing. The partial weight-bearing with crutches was allowed at 2-3 weeks after the operation in the incompetent and intact groups for these patients. The time to clinical healing was nearly six months in the incompetent and intact groups. Duration the follow-up, none of the patients experienced fixation failure.

In this controlled study, the results demonstrate that it is not so important to distinguish the integrity of the lateral wall when choosing intramedullary fixation, evaluating the quality of reduction or prognosis.

Indeed, there is a limitation in this study. The design of this study is retrospective single-center research; the patients in this study are divided into two groups according to the integrity of lateral wall. Although the baseline is comparable, there is the chance of selecting bias.

\section{Conclusions}

In conclusion, it seems that lateral wall thickness does not affect the quality of reduction and outcome in patients with intertrochanteric fracture receiving PFNA.

\section{Abbreviations \\ PFNA: Proximal femoral nail antirotation \\ HHS: Harris Hip scores \\ VAS: Visual analogue scale.}

\section{Data Availability}

The [sav] data used to support the findings of this study are available from the corresponding author upon request.

\section{Ethical Approval}

The Ethics Committee of the Xi'an Honghui Hospital approved the study (No. 2020064).

\section{Consent}

No consent was necessary.

\section{Conflicts of Interest}

The authors declare that they have no competing interests.

\section{Authors' Contributions}

Hong-Li Deng and Chao Ke contributed to the conceptualisation. Yu-Xuan Cong, Hai Huang, Bin-Fei Zhang, Ya-Hui Fu, Jin-Lai Lei, Hu Wang, Peng-Fei Wang, and Yan Zhuang contributed to the data curation. Hong-Li Deng and Chao Ke contributed to the formal analysis. Hong-Li Deng and Chao Ke contributed to the roles/writing-original draft.

\section{Acknowledgments}

This work was supported by the Social Development Foundation of Shaanxi Province (grant numbers 2017SF-050), the Natural Science Basic Research Plan in Shaanxi Province of China (2020JQ-961), and supported by the Social Development Foundation of Shaanxi Province (2019SF-120).

\section{References}

[1] A. Adeyemi and G. Delhougne, "Incidence and economic burden of intertrochanteric fracture: a medicare claims database analysis," JBJS Open Access, vol. 4, no. 1, article e0045, 2019.

[2] Y. Gotfried, "The lateral trochanteric Wall," Clinical Orthopaedics and Related Research, vol. 425, pp. 82-86, 2004.

[3] C. E. Hsu, Y. C. Chiu, S. H. Tsai, T. C. Lin, M. H. Lee, and K. C. Huang, "Trochanter stabilising plate improves treatment outcomes in AO/OTA 31-A2 intertrochanteric fractures with critical thin femoral lateral walls," Injury, vol. 46, no. 6, pp. 1047-1053, 2015.

[4] E. G. Meinberg, J. Agel, C. S. Roberts, M. D. Karam, and J. F. Kellam, "Fracture and dislocation classification compendium-2018," J Orthop Trauma, vol. 32, no. 1, Supplement 1, pp. S1-S10, 2018.

[5] A. Shetty, A. Ballal, and A. K. Sadasivan, "Dynamic hip screw with trochanteric stablization plate fixation of unstable intertrochanteric fractures: a prospective study of functional and 
radiological outcomes," Journal of Clinical and Diagnostic Research, vol. 10, pp. RC06-RC08, 2016.

[6] R. K. Gupta, K. Sangwan, P. Kamboj, S. S. Punia, and P. Walecha, "Unstable trochanteric fractures: the role of lateral wall reconstruction," International Orthopaedics, vol. 34, no. 1, pp. 125-129, 2010.

[7] A. R. Pradeep, A. KiranKumar, J. Dheenadhayalan, and S. Rajasekaran, "Intraoperative lateral wall fractures during dynamic hip screw fixation for intertrochanteric fracturesIncidence, causative factors and clinical outcome," Injury, vol. 49, no. 2, pp. 334-338, 2018.

[8] J. L. Marsh, T. F. Slongo, J. Agel et al., "Fracture and dislocation classification compendium - 2007," Journal of Orthopaedic Trauma, vol. 21, pp. S1-S6, 2007.

[9] C. Wirtz, F. Abbassi, D. S. Evangelopoulos, S. Kohl, K. A. Siebenrock, and A. Krüger, "High failure rate of trochanteric fracture osteosynthesis with proximal femoral locking compression plate," Injury, vol. 44, no. 6, pp. 751-756, 2013.

[10] H. Palm, S. Jacobsen, S. Sonne-Holm, P. Gebuhr, and Hip Fracture Study Group, "Integrity of the lateral femoral wall in intertrochanteric hip fractures: an important predictor of a reoperation," The Journal of Bone \& Joint Surgery, vol. 89, no. 3, pp. 470-475, 2007.

[11] J. Li, S. Tang, H. Zhang et al., "Clustering of morphological fracture lines for identifying intertrochanteric fracture classification with Hausdorff distance-based K-means approach," Injury, vol. 50, no. 4, pp. 939-949, 2019.

[12] T. Ma, L. Hao, P. Wen et al., "The role of preoperative computed tomography on the quality of reduction and outcomes in intertrochanteric fracture: a controlled trial," BioMed Research International, vol. 2021, Article ID 8854292, 7 pages, 2021.

[13] W. Mao, H. Ni, L. Li et al., "Comparison of Baumgaertner and Chang reduction quality criteria for the assessment of trochanteric fractures," Bone \& Joint Research, vol. 8, no. 10, pp. 502508, 2019.

[14] S. M. Chang, Y. Q. Zhang, Z. Ma, Q. Li, J. Dargel, and P. Eysel, "Fracture reduction with positive medial cortical support: a key element in stability reconstruction for the unstable pertrochanteric hip fractures," Archives of Orthopaedic and Trauma Surgery, vol. 135, no. 6, pp. 811-818, 2015.

[15] S. M. Chang, Z. Y. Hou, S. J. Hu, and S. C. du, "Intertrochanteric femur fracture treatment in Asia: what we know and what the world can learn," Orthopedic Clinics of North America, vol. 51, no. 2, pp. 189-205, 2020.

[16] P. R. Boopalan, J. K. Oh, T. Y. Kim, C. W. Oh, J. W. Cho, and W. Y. Shon, "Incidence and radiologic outcome of intraoperative lateral wall fractures in OTA $31 \mathrm{~A} 1$ and A2 fractures treated with cephalomedullary nailing," Journal of Orthopaedic Trauma, vol. 26, no. 11, pp. 638-642, 2012.

[17] Y. S. Shin, J. E. Chae, T. W. Kang, and S. B. Han, "Prospective randomized study comparing two cephalomedullary nails for elderly intertrochanteric fractures: Zimmer natural nail versus proximal femoral nail antirotation II," Injury, vol. 48, no. 7 , pp. 1550-1557, 2017. 